\title{
Intoxicação aguda por metano arsonato ácido monossódico em bovinos ${ }^{1}$
}

\author{
Gabriela N. Dantas ${ }^{2}$, Bianca P. Santarosa ${ }^{2}$, Didier Q. Cagnini ${ }^{2}$, Rodrigo M. Cavalcanti ${ }^{2}$, Simone \\ B. Chiacchio ${ }^{2}$, Roberto C. Gonçalves ${ }^{2}$, Franklin Riet-Correa ${ }^{3}$ e Alexandre S. Borges ${ }^{2 *}$
}

\begin{abstract}
Dantas G.N., Santarosa B.P., Cagnini D.Q., Cavalcanti R.M., Chiacchio S.B., Gonçalves R.C., Riet-Correa F. \& Borges A.S. 2012. [Acute poisoning by monosodium methanearsonic acid in cattle.] Intoxicação aguda por metano arsonato ácido monossódico em bovinos. Pesquisa Veterinária Brasileira 32(12):1239-1245. Departamento de Clínica Veterinária, Faculdade de Medicina Veterinária e Zootecnia, Universidade Estadual Paulista, Unesp, Campus de Botucatu, Distrito de Rubião Júnior s/n, Botucatu, SP 18618-970, Brazil. E-mail: asborges@fmvz.unesp.br

Poisoning by monosodium methanearsonic acid (MSMA) is reported in a herd of $24 \mathrm{Gi}$ rolando cows that were introduced into a pasture sprayed with the herbicide. Clinical signs were apathy, anorexia, and profuse diarrhea. Fourteen cows died and two were necropsied. Abomasal ulcers and renal congestion was observed. Main histologic lesions were multifocal, accentuated, necrotizing and hemorrhagic abomasitis and omasitis, and tubular necrosis in the kidneys. Mean arsenic concentrations in cows with clinical signs were $1.19 \pm 0.40,10.52 \pm 2.16$, and $76.06 \pm 48.37 \mathrm{ppm}$ in blood, milk, and feces, respectively. In the two necropsied cows arsenic concentrations were 25.58 and $23.85 \mathrm{ppm}$ in liver, and 28.71 and 35.94ppm in kidney, respectively. In a fetus of a cow that was necropsied, arsenic concentrations were 9.0 and $8.92 \mathrm{ppm}$ in liver and kidney, respectively. Arsenic concentration in the grass collected from the paddock sprayed with MSMA was 111.58ppm. In Brazil, the use of MSMA in the composition of herbicides is allowed only for agricultural use, not for livestock. The use of arsenic based products for livestock can lead to high mortality rates in the herd, as well as reduced production and contamination of animal products.
\end{abstract}

INDEX TERMS: Poisoning, herbicide, arsenic, monosodium methanearsonic acid, cattle.

\begin{abstract}
RESUMO. - 0 presente trabalho estudou a intoxicação acidental por arsênico em um lote de 24 vacas Girolando, as quais tiveram acesso a pasto pulverizado com herbicida à base de metano arsonato ácido monossódico (MSMA). Os bovinos apresentaram apatia, anorexia e diarreia profusa. Foram necropsiados na fazenda dois animais de 14 que morreram. Os principais achados macroscópicos foram úlceras abomasais e congestão renal. No exame microscópico, as principais lesões observadas foram abomasite e omasite necro-hemorrágica multifocal acentuada e, nos rins, necrose tubular difusa. As concentrações médias de

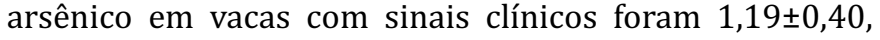

\footnotetext{
${ }^{1}$ Recebido em 6 de junho de 2012.

Aceito para publicação em 6 de setembro de 2012.

${ }^{2}$ Departamento de Clínica Veterinária, Faculdade de Medicina Veterinária e Zootecnia, Universidade Estadual Paulista (Unesp), Campus de Botucatu, Distrito de Rubião Júnior s/n, Botucatu, SP 18618-970, Brasil.

${ }^{3}$ Centro de Saúde e Tecnologia Rural, Universidade Feral de Paraíba (UFPB), Patos, PB 58700-000, Brasil. Bolsista de Produtividade Nível 2 do CNPq. *Autor para correspondência: asborges@fmvz.unesp.br
}

$10,52 \pm 2,16$ e $76,06 \pm 48,37 \mathrm{ppm}$ no sangue, leite e fezes, respectivamente. Os níveis de arsênico encontrados em dois animais necropsiados foram 25,58 e $23,85 \mathrm{ppm}$ em fígado, e 28,71 e $35,94 \mathrm{ppm}$ em rins, respectivamente. No feto de uma vaca necropsiada, os níveis de arsênico mensurados no fígado e rim foram 9,0 e 8,92ppm, respectivamente. A concentração de arsênico no capim do piquete pulverizado foi 111,58ppm. No Brasil, o uso MSMA na composição de pesticidas e herbicidas é permitido somente para uso agrícola, mas não pecuário. A utilização desse ou de outros produtos à base de arsênico na pecuária pode causar altos índices de mortalidade no rebanho, além de diminuição da produção e contaminação de produtos de origem animal.

TERMOS DE INDEXAÇÃO: Intoxicação, herbicida, arsênico, metano arsonato ácido monossódico, bovinos.

\section{INTRODUÇÃo}

0 arsênico é um metaloide pertencente ao Grupo 5A da Tabela Periódica (Meliker \& Niagru 2008) e pode ser en- 
contrado na forma de compostos orgânicos, ou inorgânicos (Graeme \& Pollack 1998), os quais, geralmente, são os relacionados com casos de intoxicação em bovinos (Oliver \& MacGregor 1957, Hatch \& Funnell 1969, Faires 2004, Valentine et al. 2007). Os compostos arsenicais inorgânicos podem ser trivalentes (arsenito) ou pentavalentes (arsenato).

A toxicidade desses compostos varia de acordo com sua solubilidade: arsenitos são os mais tóxicos, seguidos pelos arsenatos e os compostos orgânicos, por serem menos solúveis, são os menos tóxicos (Monies 1999). A absorção do arsênico pode ocorrer por via respiratória, cutânea, ou gastrointestinal. 0 arsênico é distribuído pelo organismo ligado aos eritrócitos, mas possui baixa ligação com as proteínas plasmáticas (Graeme \& Pollack 1998). A metabolização do arsênico ingerido resulta na excreção de arsênico metilado pelos rins (Kapp 2005).

Alguns produtos contendo arsênico têm sido responsáveis por casos de intoxicação em animais (Neiger et al. 2004), mais comumente, em bovinos e felinos, por ingestão de alimentos contaminados (Buck et al. 1973). Quando o arsênico se liga aos elementos celulares, ocorre a inibição da respiração mitocondrial e desacoplamento da fosforilação oxidativa, entre outros efeitos (Rehner 2003). 0 resultado dessa perturbação no metabolismo celular tem efeitos sobre o encéfalo, pulmão, rim, fígado e mucosa do trato gastrointestinal. Além disso, o arsênico também causa aumento da permeabilidade capilar sistêmica (Palmer 1972). 0 metano arsonato ácido monossódico (MSMA) é um arsênico orgânico, cujos sinais clínicos mais comuns de intoxicação são semelhantes aos descritos para os arsênicos inorgânicos (Palmer 1972) e incluem diarreia, apatia e anorexia (Buck et al. 1973). Abomasite hemorrágica e enterite são as principais anormalidades descritas em bovinos intoxicados com arsênico inorgânico (Monies 1999).

A intoxicação por arsênico inorgânico em bovinos foi, anteriormente, descrita na literatura internacional por Oliver \& MacGregor (1957), Hatch \& Funnell (1969), Selby et al. (1977), McClanahan et al. (2003), Faires (2004), Valentine et al. (2007) e, por arsênico orgânico, por Neiger et al. (2004). No Brasil, foram descritos dois surtos de intoxicação por arsênico em bovinos, um no Paraná e outro no Rio de Janeiro; alguns animais morreram em forma aguda e outros apresentaram abaulamento da parede do abdome do lado direito, próximo ao umbigo, que rompia a pele dentro de 4-6 semanas e, frequentemente, seguia-se o prolapso da parede do abomaso (Döbereiner et al. 1967). Na década de 1980, carrapaticidas à base de trióxido de arsênico (inorgânico) eram utilizados no Brasil e foram mencionados como causadores de intoxicação em bovinos (Riet-Correa 2007). Apesar dessas descrições, os dados clínicos e laboratoriais sobre a intoxicação por arsênico são escassos; portanto, o objetivo desse trabalho foi descrever a intoxicação acidental aguda por arsênico orgânico em bovinos.

\section{MATERIAL E MÉTODOS}

Intoxicação acidental aguda por arsênico em bovinos leiteiros, provenientes do município de Avaré/SP, localizado na região centro-oeste paulista, foi estudada pelo Serviço de Clínica Médica de
Grandes Animais (CMGA) da Faculdade de Medicina Veterinária e Zootecnia (FMVZ), da Universidade Estadual Paulista "Julio de Mesquita Filho" (Unesp), Campus de Botucatu, no ano de 2011.

Para o estudo clínico-laboratorial foram avaliados os sinais clínicos, hemograma e bioquímica sérica de dois bovinos (bovinos 1 e 2) com histórico de apatia, anorexia e diarreia profusa, encaminhados à CMGA-FMVZ/Unesp, cinco dias após a exposição ao herbicida. Foram avaliados atitude, funções vitais (coloração de mucosas, frequências cardíaca e respiratória, temperatura, grau de hidratação e movimentação ruminal) e o escore corporal. Amostras de sangue total foram colhidas por punção de veia jugular, em tubos com e sem anticoagulante, para realização do hemograma e bioquímica sérica (função renal e hepática, níveis de proteínas e creatina quinase), respectivamente. Amostras de sangue total foram colhidas em tubo com heparina e encaminhadas para determinação de concentração de arsênico.

Foi realizada visita à propriedade rural, oito dias após a exposição dos bovinos ao herbicida, para colheita de amostras de sangue total (com heparina), leite e fezes de outras dez vacas para a determinação dos níveis de arsênico, sendo cinco delas do lote afetado e cinco normais, de outro lote que não teve acesso ao pasto pulverizado com arsênico suspeito de intoxicar os bovinos. Além disso, amostras de capim do piquete pulverizado também foram encaminhadas para determinação da concentração de arsênico.

Ainda na propriedade rural, foram realizadas necropsias de duas vacas imediatamente após o óbito (Bovinos 3 e 4), do lote que teve acesso ao pasto pulverizado e de um feto (terço final de gestação) de uma dessas vacas. Foram colhidas amostras de vários órgãos para exame histopatológico e amostras de fígado e rim destes dois bovinos e do feto necropsiados foram encaminhadas para determinação das concentrações de arsênico.

A atividade sérica das enzimas do exame bioquímico foi determinada pelo método cinético com reagentes bioquímicos comerciais (Analisa ${ }^{\circledR}$ ) e a leitura, efetuada em espectrofotômetro (Celm SB-190). O hemograma foi determinado em contador automático de células (Cell-Dyn 3500R Abbot). A proteína total e o fibrinogênio foram determinados por refratometria, segundo método descrito por Jain (1993). A determinação das concentrações de arsênico em amostras de fígado, rins, fezes e capim foi feita utilizando-se matéria seca. Todas as determinações de concentração de arsênico foram realizadas pelo Centro de Análises Toxicológicas da Unesp, Campus de Botucatu (CEATOX), pela técnica de espectrometria por absorção atômica, no aparelho GBC AA 932 Plus. As médias dos grupos doente e clinicamente normal foram analisadas pelo Teste "T" não pareado com correção de Welch.

\section{RESULTADOS}

\section{Epidemiologia, sinais clínicos, tratamento e evolução}

A propriedade apresentava plantel de 83 vacas da raça Girolanda, em sistema de manejo semi-intensivo, com média de 15 litros de leite/animal/dia produzidos em duas ordenhas diárias. Os bovinos em lactação eram suplementados com ração (18\% de energia, à base de caroço de algodão), duas vezes ao dia.

Um dos dois lotes de animais, composto por 24 vacas em lactação, foi levado ao piquete de $1.200 \mathrm{~m}^{2}$ (capim Jiggs, cultivar de Cynodon dactylon) pulverizado com herbicida contendo arsênico orgânico MSMA, aproximadamente, 1 hora após a aplicação do produto. 0 período de exposição das vacas no piquete pulverizado foi de 10 horas, quando então foram encaminhadas para a sala de ordenha e os 24 animais do lote exposto apresentavam diarreia líquida e fé- 
tida, além de queda na produção de leite de 15L para 3L/ animal, apatia, anorexia, sialorreia, tremores musculares e desidratação. No decorrer do dia, essas vacas permaneciam em decúbito esternal por período prolongado e com intensa vocalização. Após a ordenha, as vacas foram levadas a um piquete não pulverizado com o herbicida. Aproximadamente 48 horas após o início dos sinais clínicos, instituiu-se tratamento com $30 \mathrm{~g}$ de tiossulfato de sódio, via oral, dose única, porém sem sucesso. A utilização do MSMA já havia sido realizada na propriedade em outras ocasiões, mas sem relatos de anormalidades clínicas prévias.

Ao longo dos 15 dias subsequentes à intoxicação, $58,33 \%(14 / 24)$ dos animais do lote morreram. 0 primeiro óbito ocorreu 34 horas após a exposição ao pasto contaminado e $64,28 \%$ (9/14) dos óbitos, até o sexto dia. A última das 14 vacas morreu 21 dias após a exposição ao herbicida. Não foram observadas anormalidades nos animais da propriedade que não tiveram acesso ao piquete pulverizado.

Os Bovinos 1 e 2 apresentavam bom estado nutricional $(\mathrm{ECC}=3 / 5)$, apatia, palidez da mucosa ocular, desidratação (8\%), hipotermia $\left(\mathrm{T} \leq 36^{\circ} \mathrm{C}\right)$, taquipnéia $(\mathrm{FR} \geq 60 \mathrm{mpm})$, taquicardia ( $\mathrm{FC} \geq 100 \mathrm{bpm}$ ), hipotonia ruminal ( $\mathrm{MR} \leq 2 / 5$ minutos) e anúria. Os animais vocalizavam e apresentavam decúbito intermitente. Havia crepitação grossa pulmonar difusa e presença de diarreia líquida fétida, de coloração marrom escura, sem estrias de sangue. Diariamente, os animais receberam transfaunação com cerca de $3 \mathrm{~L}$ de líquido ruminal e hidratação por via oral com 15 litros de água morna adicionada de probiótico, sal mineral, suplemento energético e vitamínico. Além disso, foi administrado flunixim meglumine $(2,2 \mathrm{mg} / \mathrm{kg}$, intramuscular) e $30 \mathrm{~g}$ de tiossulfato de sódio por via oral, por quatro dias consecutivos, conforme proposto por Buck et al. (1973) e Garry (2008).

Após dois dias de acompanhamento, o Bovino 1 apresentou piora do quadro clínico, com diarreia sanguinolenta e morte. Após uma semana de internação, o Bovino 2 apresentou melhora do estado geral, retorno dos movimentos ruminais, mas ainda apresentava hiporexia e fezes pastosas. Esse bovino retornou à propriedade, tendo sobrevivido à exposição ao arsênico.

\section{Exames complementares}

0 resultado do hemograma, bioquímica sérica e níveis de arsênico sanguíneo dos Bovinos 1 e 2 estão apresentados no Quadro 1.

As concentrações de arsênico no sangue, leite e fezes das vacas da propriedade estão apresentadas no Quadro 2. Foram observadas diferenças significativas nas médias dos grupos de animais doentes e clinicamente normais nas amostras de sangue $(\mathrm{p}=0,0081)$, leite $(\mathrm{p}=0,0083)$ e fezes $(\mathrm{p}=0,0488)$.

No fígado das vacas necropsiadas, na propriedade rural, os valores de arsênico encontrados foram 25,58ppm e $23,85 \mathrm{ppm}$. Já, nos rins, os valores foram $28,71 \mathrm{ppm}$ e $35,94 \mathrm{ppm}$. 0 fígado e os rins do feto apresentaram valores de arsênico de 9,0ppm e 8,92ppm, respectivamente. A concentração de arsênico no capim coletado do piquete pulverizado foi de 111,58ppm.
Quadro 1. Hemograma, bioquímica sérica e níveis de arsênico sanguíneo na intoxicação aguda por metano arsonato ácido monossódico em bovinos

\begin{tabular}{|c|c|c|c|}
\hline & Bovino 1 & Bovino 2 & $\begin{array}{l}\text { Valores de referência } \\
\text { (Kaneko et al. 1997) }\end{array}$ \\
\hline Neutrófilos & $6400 / \mu \mathrm{L}$ & $6100 / \mu \mathrm{L}$ & 600 a $4000 / \mu \mathrm{L}$ \\
\hline Monocitos & $2100 / \mu \mathrm{L}$ & $1300 / \mu \mathrm{L}$ & 25 a $840 / \mu \mathrm{L}$ \\
\hline Hemácias & $9,44 \times 10^{6} / \mu \mathrm{L}$ & $10,75 \times 10^{6} / \mu \mathrm{L}$ & 5 a $10 \times 10^{6} / \mu \mathrm{L}$ \\
\hline Hemoglobina & $14,7 \mathrm{~g} / \mathrm{dL}$ & $18,3 \mathrm{~g} / \mathrm{dL}$ & 8 a $153 g / d L$ \\
\hline Hematócritos & $44 \%$ & $55 \%$ & 24 a $46 \%$ \\
\hline PPT* & $9,5 \mathrm{~g} / \mathrm{dL}$ & $11,3 \mathrm{~g} / \mathrm{dL}$ & 7 a $8,5 \mathrm{~g} / \mathrm{dL}$ \\
\hline Globulina & $6,6 \mathrm{~g} / \mathrm{dL}$ & $8,1 \mathrm{~g} / \mathrm{dL}$ & 3 a $3,48 \mathrm{~g} / \mathrm{dL}$ \\
\hline GGT* & $24,9 \mathrm{UI} / \mathrm{L}$ & $28,5 \mathrm{UI} / \mathrm{L}$ & 6,1 a $17,4 \mathrm{UI} / \mathrm{L}$ \\
\hline AST* & 96,1UI/L & $245,9 \mathrm{UI} / \mathrm{L}$ & 78 a $132 \mathrm{UI} / \mathrm{L}$ \\
\hline Creatinina & $1,87 \mathrm{mg} / \mathrm{dL}$ & $6,28 \mathrm{mg} / \mathrm{dL}$ & 1 a $2 \mathrm{mg} / \mathrm{dL}$ \\
\hline Ureia & $80 \mathrm{mg} / \mathrm{dL}$ & $194 \mathrm{mg} / \mathrm{dL}$ & 42,8 a $64,2 \mathrm{mg} / \mathrm{dL}$ \\
\hline Bilirrubina total & $0,42 \mathrm{mg} / \mathrm{dL}$ & $0,13 \mathrm{mg} / \mathrm{dL}$ & 0,01 a $0,47 \mathrm{mg} / \mathrm{dL}$ \\
\hline Bilirrubina indireta & $0,2 \mathrm{mg} / \mathrm{dL}$ & $0,04 \mathrm{mg} / \mathrm{dL}$ & 0 a $0,03 \mathrm{mg} / \mathrm{dL}$ \\
\hline Bilirrubina direta & $0,22 \mathrm{mg} / \mathrm{dL}$ & $0,09 \mathrm{mg} / \mathrm{dL}$ & 0,04 a $0,44 \mathrm{mg} / \mathrm{dL}$ \\
\hline Albumina & $3,2 \mathrm{~g} / \mathrm{dL}$ & $2,9 \mathrm{~g} / \mathrm{dL}$ & 3,03 a $3,55 \mathrm{~g} / \mathrm{dL}$ \\
\hline Arsênico & $3,49 \mathrm{ppm}$ & 2,78ppm & \\
\hline
\end{tabular}

* PPT = proteína plasmática total, GGT = gamaglutamiltransferase, AST = aspartatoaminotransferase.

Quadro 2. Valores de arsênico no sangue (ppm), leite (ppm) e fezes (ppm) de cinco bovinos acidentalmente intoxicados por arsênico e cinco bovinos clinicamente normais*

\begin{tabular}{cccccccc}
\hline \multirow{2}{*}{ Animal } & \multicolumn{3}{c}{ Doentes } & & \multicolumn{3}{c}{ Clinicamente normais } \\
\cline { 2 - 3 } \cline { 6 - 8 } & Sangue & Leite & Fezes & & Sangue & Leite & Fezes \\
\hline 1 & 1,09 & 8,70 & 16,60 & & 0,28 & 1,80 & 5,10 \\
2 & 1,06 & 8,90 & 128,4 & & 0,17 & 0,30 & 3,40 \\
3 & 1,89 & 9,90 & 68,90 & & 0,40 & 4,90 & 20,40 \\
4 & 1,07 & 14,00 & 121,50 & & 0,18 & 2,00 & 20,40 \\
5 & 0,84 & 11,10 & 44,90 & & 0,37 & 3,20 & 22,50 \\
Média & $1,19^{\mathrm{a}}$ & $10,52^{\mathrm{a}}$ & $76,06^{\mathrm{a}}$ & & $0,28^{\mathrm{b}}$ & $2,44^{\mathrm{b}}$ & $14,36^{\mathrm{b}}$ \\
Desvio padrão & 0,40 & 2,16 & 48,37 & & 0,10 & 1,71 & 9,28
\end{tabular}

* Letras diferentes representam diferença estatística entre os grupos, para o mesmo material analisado.

\section{Achados macroscópicos}

Os animais apresentavam mucosas ocular e vulvar congestas e o rúmen continha grande quantidade de conteúdo alimentar composto, basicamente, por volumoso. 0 omaso apresentava avermelhamento da mucosa com áreas de erosão discretas. No abomaso e na porção inicial do intestino delgado, havia conteúdo alimentar hemorrágico e úlceras multifocais com centro necrótico e bordas avermelhadas, entre 0,3 e $3 \mathrm{~cm}$ de diâmetro e mais extensas nas plicas do abomaso (Fig.1). Esse quadro foi caracterizado por abomasite e enterite proximal necrotizante acentuada. Além disso, foi observada ausência de conteúdo alimentar e moderada quantidade de muco, coágulos e restos de mucosa nas porções distais de intestino delgado e grosso. Nos rins, a região medular apresentava coloração vermelho-escura difusa (Fig.2). 0 pulmão estava pesado, armado e apresentava moderada quantidade de líquido espumoso claro em brônquios principais (edema pulmonar). Foram evidenciadas petéquias e sufusões subendocárdicas e subepicárdicas nos átrios e ventrículos.

\section{Achados microscópicos}

No rúmen, a microscopia revelou degeneração hidrópica multifocal discreta do epitélio com áreas focais de 


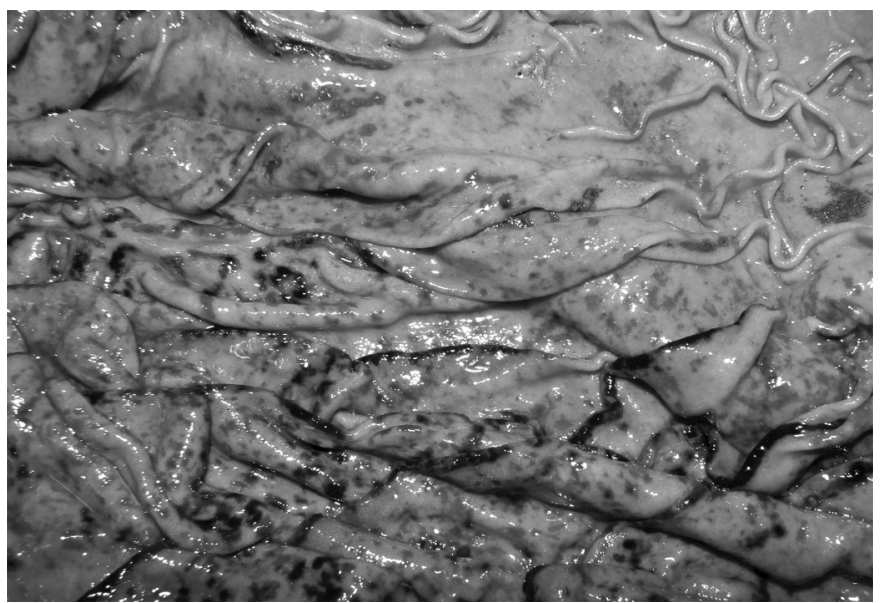

Fig.1. Avermelhamento da mucosa do abomaso associado à presença de inúmeras úlceras com distribuição multifocal e aleatória, na intoxicação por arsênico em bovino.

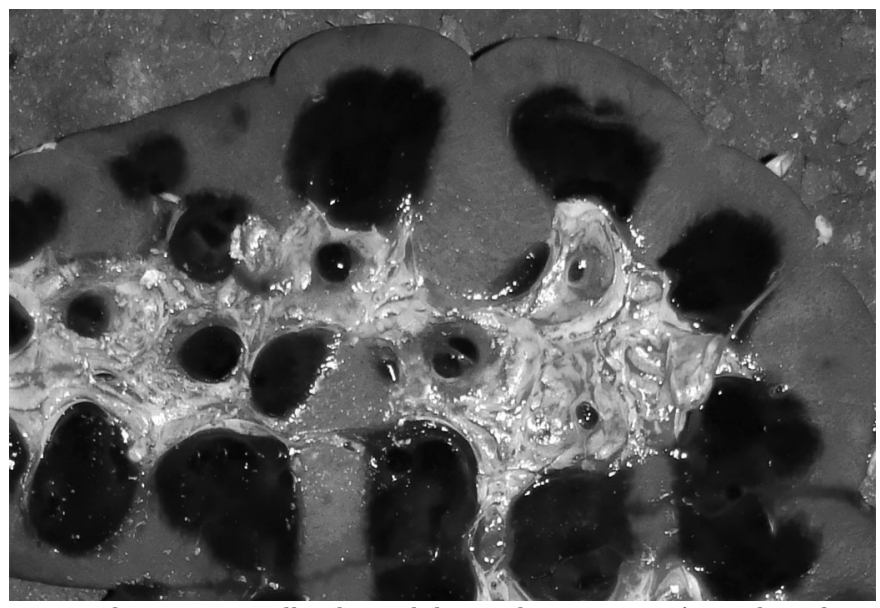

Fig.2. Coloração vermelha da medula renal em consequência da nefrose hemorrágica difusa, na intoxicação por arsênico.



Fig.3. Abomasite necro-hemorrágica, na intoxicação de bovino por arsênico. HE, obj.10x. Barra: $200 \mu \mathrm{m}$.

ulceração. No retículo, observou-se degeneração hidrópica acentuada e necrose moderada do epitélio. No omaso, verificou-se degeneração hidrópica e necrose multifocal do epitélio. $\mathrm{O}$ abomaso apresentava abomasite necro-hemorrágica multifocal acentuada (Fig.3). No intestino delgado,

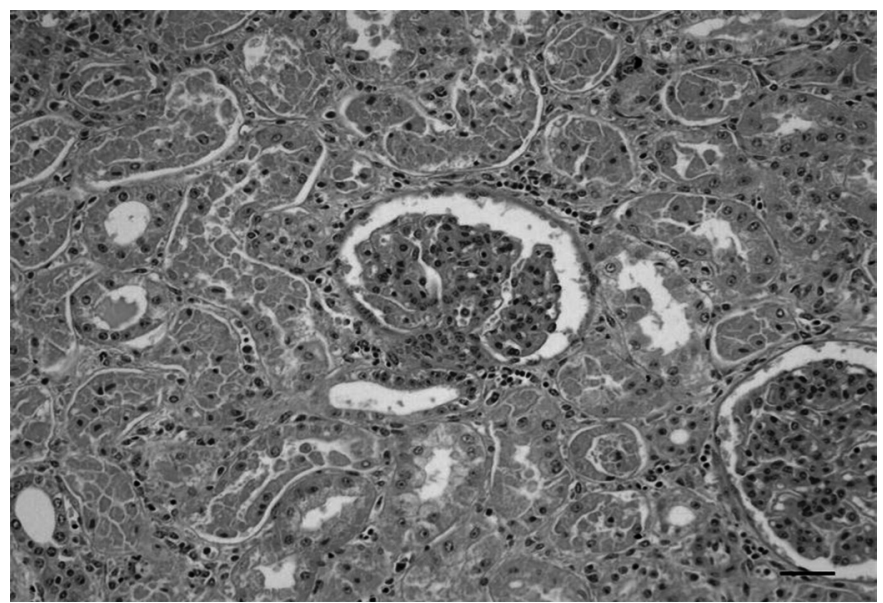

Fig.4. Córtex renal com necrose tubular aguda, difusa, acentuada, na intoxicação de bovino por arsênico. HE, obj.20x. Barra: $100 \mu \mathrm{m}$

havia necrose focal do topo das vilosidades com presença de infiltrado de células mononucleares. Havia também nefrose multifocal a coalescente acentuada na região cortical (Fig. 4) e nefrose hemorrágica difusa acentuada na medular, ocasionalmente, associada a severo infiltrado polimorfonuclear, principalmente, na junção corticomedular. Verificou-se a presença de áreas multifocais de hemorragias discretas subendocárdicas e subepicárdicas. Os demais órgãos não apresentaram alterações histológicas.

\section{DISCUSSÃO}

0 uso comercial de arsênico está diminuindo desde a década de 1960 (Neiger et al. 2004). Atualmente, é uma das causas de intoxicação menos frequentes em rebanhos devido ao seu banimento de quase todas as fases das atividades agropecuárias (Radostits et al. 2007). 0 arsênico presente nos produtos de uso agrícola pertence à família dos compostos arsenicais orgânicos alifáticos (Plumlee 2006). No Brasil, o Ministério da Agricultura, Pecuária e Abastecimento permite o uso do arsênico na composição de alguns pesticidas, herbicidas de utilização agrícola e tintas (Resolução - RDC no 135 de 17/05/02). Neste estudo, o herbicida (MSMA) pulverizado na pastagem foi a fonte de arsênico. Apesar do uso do MSMA ser permitido para agricultura, este composto foi utilizado nos pastos da propriedade para controle de ervas daninhas. No entanto, não foram relatados casos de intoxicação anteriores na propriedade, pois os animais eram colocados no pasto pulverizado somente após a secagem do produto, diferente do presente relato onde os animais foram encaminhados ao pasto 1 hora após a pulverização.

Os sinais clínicos observados nos animais deste trabalho foram sialorreia, vocalização, diarreia profusa, letargia, depressão, anorexia, taquipneia, queda da produção de leite, apatia, decúbito por períodos prolongados, tremores musculares e desidratação, achados similares aos descritos na intoxicação por arsênicos inorgânicos por Monies (1999) e Faires (2004). McClanahan et al. (2003) relataram um surto da intoxicação por arsênico inorgânico em gado de corte e descreveram odor arsenical no hálito dos animais intoxi- 
cados; enquanto Faires (2004) citou bradicardia e ataxia, sinais que não foram observados nos animais deste estudo. Monies (1999) relatou que o óbito, frequentemente, ocorre entre 24 e 48 horas, diferente do que foi observado no presente estudo, onde somente uma das 14 mortes observadas ocorreu em menos de 48 horas e 64,28\% (9/14) dos óbitos ocorreram entre o $2^{\circ}$ e o 6ํㅜㄹ dias após a ingestão do alimento contaminado. Essa diferença ocorre, provavelmente, em consequência dos níveis de arsênico da dieta. Além disso, o período de exposição ao alimento contaminado e o tipo de arsênico também podem influenciar a apresentação e a evolução do quadro de intoxicação.

Taquicardia, aumento no tempo de preenchimento capilar, diminuição da pressão de pulso, da elasticidade da pele e da produção de urina evidenciaram quadro de desidratação, confirmado pelos achados laboratoriais de hiperproteinemia e policitemia (Kaneko et al. 1997). 0 fibrinogênio, útil indicador de inflamação em bovinos (Morris \& Johnston 2006), apresentou-se elevado nas amostras das duas vacas internadas na CMGA. O leucograma não apresentou leucocitose, porém mostrou neutrofilia e monocitose (Kaneko et al. 1997). A toxicidade do arsênico provoca alterações no sistema gastrointestinal, com consequente manifestação clínica de diarreia, explicação plausível para a neutrofilia nos exames dos animais afetados. A contagem de monócitos não é altamente responsiva à doença inflamatória em grandes animais; portanto, não se constituiu em componente útil do leucograma (Morris 2006).

Os dois bovinos apresentaram azotemia decorrente da toxicidade renal causada pelo arsênico. Apenas uma das vacas apresentou elevação do nível de AST, que pode ser explicado pela lesão muscular devido ao decúbito prolongado. Nas amostras analisadas dos bovinos intoxicados não houve aumento de GGT e FA. O produto à base de arsênico não apresentou hepatoxicidade suficiente para elevar essas enzimas no momento da análise. Os animais afetados não apresentaram icterícia, embora uma vaca apresentasse aumento discreto nos valores das bilirrubinas. $\mathrm{O}$ aumento de globulina evidenciado pode estar relacionado com a marcante inflamação tecidual que ocorre, principalmente, em trato gastrointestinal (Kaneko et al. 1997).

Trabalhos envolvendo casos de intoxicação de bovinos por arsênico descreveram os níveis do elemento em fígado, rim, ingesta, urina, leite, pastagem, solo e água (Oliver \& MacGregor 1957, Hatch \& Funnell 1969, Selby et al. 1977, Hopkirk 1987, McClanahan et al. 2003, Faires 2004, Neiger et al. 2004, Valentine et al. 2007). Os estudos acima descreveram intoxicação por arsênico inorgânico, com exceção de Neiger et al. (2004) que relataram intoxicação por arsênico orgânico. No presente estudo, além de fígado, rim, leite e pastagem, também foram determinados os níveis de arsênico no sangue e fezes.

Hatch \& Funnell (1969) encontraram média de 35,7ppm do elemento na ingesta de 21 bovinos que morreram com sinais de intoxicação por arsênico. Hopkirk (1987) relatou surto de intoxicação em 200 vacas; cuja forragem continha $132 \mathrm{ppm}$ de arsênico, valor semelhante ao encontrado no piquete pulverizado com MSMA do presente relato.
Os Bovinos 1 e 2 apresentaram valores de 3,49 e 2,78ppm de arsênico sanguíneo 120 horas após a exposição, enquanto os animais da fazenda, do mesmo lote, apresentaram média de 1,19ppm de arsênico no sangue 156 horas após a exposição. Essa diferença pode ser explicada pelo fato dessas amostras terem sido colhidas com intervalo de 36 horas, intervalo em que houve diminuição da concentração de arsênico sanguíneo. É interessante observar que relatos prévios de intoxicação acidental de bovinos por arsênico não descreveram a concentração sanguínea desse elemento nos animais intoxicados, o que dificulta qualquer análise comparativa associada. Como o arsênico é naturalmente encontrado nas plantas e no solo, traços desse microelemento estão presentes nos tecidos da maioria dos animais (Plumlee 2006). Alonso et al. (2000) mediram a presença de poluentes ambientais em animais clinicamente normais e encontraram 0,00292ppm de arsênico em sangue total. Valor que foi menor que $0,28 \mathrm{ppm}$ encontrado nos animais clinicamente normais deste trabalho e, provavelmente, associado com exposições prévias pelo uso do arsênico em pastos da propriedade. Ressalta-se que animais podem apresentar altos níveis de arsênico em amostras teciduais, como foi constatado neste trabalho, mesmo sem apresentar sintomatologia clínica.

A mensuração do elemento em fígado e rins é de extrema importância, pois retrata a intoxicação, já que esses órgãos acumulam as mais altas concentrações de metais pesados circulantes no organismo (Alonso et al. 2000). Segundo Buck et al. (1973) e Faires (2004), níveis de arsênico entre 10 e 15ppm no fígado, acompanhados de sinais clínicos, são patognomônicos de intoxicação aguda pelo elemento, o que corrobora com os achados deste trabalho, onde os valores encontrados no fígado das vacas necropsiadas foram cerca de duas vezes mais elevados que os valores relatados acima. Os níveis de arsênico renal e hepático descritos em casos anteriores de intoxicação em bovinos, na matéria úmida, variaram entre 6,18 e $60 \mathrm{ppm}$ e 12,8 e 24ppm, respectivamente (Buck et al. 1973, McClanahan et al. 2003, Valentine et al. 2007). Alonso et al. (2000) mensuraram média, em matéria úmida, 0,0102ppm no fígado e 0,0152ppm nos rins de animais sem sintomatologia, porém expostos ao arsênico devido a poluição ambiental.

Apesar de não terem sido encontrados na literatura níveis de arsênico teciduais em fetos de vacas gestantes intoxicadas para comparação, os níveis de arsênico encontrados no presente relato foram 18 vezes maiores que o limite tolerável (0,5ppm) em tecidos bovinos (Buck et al. 1973, Bahri \& Romdane 1991, Monies 1999).

Os níveis de arsênico no leite de animais clinicamente normais e de animais intoxicados foram muito elevados, em decorrência do manejo adotado na propriedade. O MSMA, tipo de arsênico orgânico responsável pela intoxicação dos animais neste trabalho, é um ânion, em pH neutro (Barra et al 2000). Assim, maiores níveis de arsênico no leite que no sangue poderiam ser explicados pela afinidade dos ânions de arsênico pelos cátions de cálcio presentes no leite, estabelecendo uma via de excreção desse metaloide, por isso se devem controlar seus níveis em alimentos para consumo humano (Gomez-Caminero et al. 2001). 
De acordo com Monies (1999), as principais lesões macroscópicas são abomasite hemorrágica, inflamação do intestino delgado, ulceração da mucosa omasal, fígado com coloração acinzentada e, ocasionalmente, úlceras em mucosa oral. No presente estudo, foram encontradas alterações macroscópicas semelhantes. Além disso, Faires (2004) relatou hemorragia em meninge e pericárdio, e lesões erosivas em abomaso, duodeno, cólon e reto, o que, com exceção da alteração de meninge, corrobora com os achados deste trabalho. Oliver \& Macgregor (1957) ressaltaram que lesões profusas em trato gastrointestinal são os principais achados de necropsia na intoxicação por arsênico. Assim como o descrito por Neiger et al. (2004), os animais necropsiados neste estudo apresentaram o rúmen totalmente preenchido por conteúdo alimentar. Microscopicamente, os achados de gastroenterite hemorrágica e as alterações em túbulos renais estão de acordo com o descrito na literatura em casos de intoxicação por arsênico em vacas (Brown et al. 2007, Maxie \& Newman 2007), no entanto a necrose de linfonodos da cadeia mesentérica descrita por Neiger et al. (2004) não foi observada neste trabalho. Nos casos que a morte ocorre dentro das primeiras horas de intoxicação as alterações macro e microscópicas podem ser ausentes ou mínimas, podendo ser evidenciado apenas edema e congestão discretos da mucosa abomasal (Maxie \& Youssef 2007).

0 tratamento da intoxicação por arsênico depende do tipo e da gravidade dos sinais clínicos. Nos casos agudos é pouco eficaz devido às grandes quantidades ingeridas e ao tempo decorrido entre a ingestão e o início das manifestações clínicas (Radostits et al. 2007). O tiossulfato de sódio é um antídoto frequentemente utilizado por não ser tóxico e seguro em grandes animais (Buck et al. 1973). Esse foi o produto empregado no tratamento das vacas intoxicadas, por ser mais acessível no mercado e ter menor custo.

Os compostos que contêm enxofre são teoricamente os melhores antídotos, dos quais o 2,3 dimercaptopropanol ou dimercaprol (BAL) é o produto utilizado em casos de intoxicação por composto arsenical trivalente, inorgânico ou orgânico alifático (Bahri \& Romdane 1991, Plumlee 2006), no entanto esse elemento é pouco eficaz nos casos de envenamento por sais inorgânicos (Radostits et al. 2007). 0 uso, entretanto, desse produto é restrito em animais de produção devido ao alto custo e disponibilidade no mercado (Monies 1999).

0 ácido etilenodiaminotetracético (EDTA) não é eficaz porque esse quelante remove apenas o arsênico extracelular, não o intracelular, responsável pelos sinais clínicos. Em humanos, a D-penicilamina é eficaz, porém seu uso é anti-econômico em animais de produção. 0 ácido dimercaptosuccínico (DMSA) é um produto mais recente, menos tóxico que o BAL, e está disponível para uso em humanos e pequenos animais, podendo ser considerado futuro tratamento da intoxicação por arsênico (Plumlee 2006).

A terapia de suporte também deve ser instalada, com reposição hidro-eletrolítica, lavagem gástrica e transfaunação, assim como instituído neste caso (Buck et al. 1973). Podem-se utilizar adsorventes, como carvão ativado (1-4g/ $\mathrm{kg}$, via oral), e posteriormente laxantes osmóticos, como sulfato de magnésio. Diversos produtos são usados para precipitar o arsênico na luz intestinal. 0 hidrato férrico é mais comumente usado, porém tem pouco efeito evidente na evolução da doença (Radostits et al. 2007). A adoção de terapia anti-inflamatória nos animais atendidos teve como objetivo promover analgesia e controlar a inflamação. McClanahan et al. (2003) citaram o prognóstico ruim em casos de intoxicação aguda por arsênico, a inviabilidade econômica e baixa efetividade do tratamento em grandes animais.

Além disso, recomendou-se interromper a prática de pulverização com o herbicida nos piquetes destinados aos animais para evitar novos casos clínicos, ou mesmo a exposição de bovinos pela ingestão de arsênico, como poluente ambiental.

\section{CONCLUSÕES}

A intoxicação por arsênico deve ser incluída no diagnóstico diferencial de doenças do sistema digestivo que apresentem diarreia e lesões degenerativas e necróticas, sobretudo no omaso e abomaso.

A confirmação da intoxicação deve ser realizada mediante análise toxicológica por dosagem do metal no sangue total, fígado e rins.

$\mathrm{O}$ uso de produtos à base de arsênico deve ser evitado, pois pode levar a altos índices de mortalidade no rebanho, além de diminuição da produção e contaminação de produtos de origem animal.

Agradecimentos.- Ao graduando Carlos Ramires Neto e aos médicos veterinários Alex Cerqueira Carosi e Ana Cláudia Gorino, pela ajuda na colheita das amostras; ao pós-graduando Peres Ramos Badial, pela análise estatística; e ao farmacêutico-bioquímico Alaor Aparecido Almeida, pela análise toxicológica.

\section{REFERÊNCIAS}

Alonso M.L., Benedito J.L., Miranda M., Castillo C., Hernandez J. \& Shore R.F. 2000. Arsenic, cadmium, lead, copper and zinc in cattle from Galicia, NW Spain. Sci. Total Environ. 246:237-248.

Bahri L.E. \& Romdane S.B. 1991. Arsenic poisoning in livestock. Vet. Human Toxicol. 33:259-264.

Barra C.M., Santelli R.E., Abrão J.J. \& Guardia M. 2000. Especiação de arsênio: uma revisão. Quím. Nova 23:58-70.

Brown C.C., Baker D.C. \& Barker I.K. 2007. Alimentary system, p.59-132. In: Maxie M.G. (Ed.), Jubb, Kennedy, Palmer's Pathology of Domestic Animals. $5^{\text {th }}$ ed. Saunders Elsevier, Ontario.

Buck W.B., Osweiler G.D. \& Van Gelder G.A. 1973. Clinical and diagnostic veterinary toxicology, p.281-288. $2^{\text {nd }}$ ed. Kendall/Hunt Publishing Company, USA.

Döbereiner J., Camargo W.V., Valente F.T., Alencar Filho R.A., Canella C.F. \& Tokarnia C.H. 1967. Perfuração do abomaso e da parede abdominal na intoxicação de bovinos por arsênio. Pesq. Agropec. Bras. 2:293-299.

Faires M.C. 2004. Inorganic arsenic toxicosis in a beef herd. Can. Vet. J. 45:329-331.

Garry F. 2008. Miscellaneous toxicities and deficiences, p.640-650. In: Divers T.J. \& Peek S.F. (Eds), Rebhun's Diseases of Dairy Cattle. $2^{\text {nd }}$ ed. Elsevier, St Louis.

Gomez-Caminero A., Howe P., Hughes M., Kenyon E., Lewis D.R., Moore M., Ng J., Aitio A. \& Becking G. 2001. Arsenic and Arsenic Compounds. $2^{\text {nd }}$ ed. World Health Organization, Geneva. 512p.

Graeme K.A. \& Pollack Jr. C.V. 1998. Heavy metal toxicity. Part 1. Arsenic and mercury. J. Emerg. Med. 16:45-56. 
Hatch R.C. \& Funnell H.S. 1969. Inorganic arsenic levels in tissues and ingesta of poisoned cattle: An eight-year survey. Can. Vet. J. 10:117-120.

Hopkirk R.G. 1987. Arsenic poisoning in a dairy cattle from naturally occurring arsenic pyrites. N. Z. Vet. Journal 35:170-172.

Jain N.C. 1993. Essentials of Veterinary Hematology. Lea and Febiger, Philadelphia.

Kaneko J.J., Harvey J.W. \& Bruss M.L. 1997. Clinical Biochemistry of Domestic Animals. $5^{\text {th }}$ ed. Academic Press, San Diego, 932p.

Kapp R. 2005. Arsenic, p.168-171. In: Anderson B., Peyster A., Gad S.C., Hakkinen P.J.B., Kamrin M., Locey B., Mehendade H.M., Pope C., Shugart L. \& Wexler P. (Eds), Encyclopedia of Toxicology. Vol.1. Academic Press, San Diego.

Maxie M.G. \& Youssef S. 2007. Nervous system, p.369. In: Maxie M.G. (Ed.), Jubb, Kennedy, Palmer's Pathology of Domestic Animals. $5^{\text {th }}$ ed., Saunders Elsevier, Oxford.

Maxie M.G. \& Newman S.J. 2007. Urinary system, p.469. In: Maxie M.G. (Ed.), Jubb, Kennedy, Palmer's Pathology of Domestic Animals. $5^{\text {th }}$ ed. Saunders Elsevier, Ontario.

McClanahan S., Ames T. \& Murphy M. 2003. Arsenic poisoning of beef cattle herd. Minnesota Beef Cow, Calf Days, p.83-87.

Meliker J.R. \& Nriagu J.O. 2008. Arsenic, p.233-238. In: Anderson B., Peyster A., Gad S.C., Hakkinen P.J.B., Kamrin M., Locey B., Mehendade H.M., Pope C., Shugart L. \& Wexler P. (Eds), Encyclopedia of Toxicology. Vol.1. Academic Press, San Diego.

Monies B. 1999. Arsenic poisoning in cattle. In Practice 21:602-607.

Morris D.D. 2006. Alterações no leucograma, p.420-426. In: Smith B.P. (Ed.), Medicina Interna de Grandes Animais. 3르 ed. Manole, Barueri.
Morris D.D. \& Johnston J.K. 2006. Alterações nas proteínas do sangue, p.427-433. In: Smith B.P. (Ed.), Medicina Interna de Grandes Animais. 3르. Manole, Barueri.

Neiger R., Nelson N., Miskimins D., Caster J. \& Caster L. 2004. Bovine arsenic toxicosis. J. Vet. Diagn. Invest. 16:436-438.

Oliver W.T. \& MacGregor K.L. 1957. Lead arsenate poisoning in a dairy cattle herd. Can. J. Comp. Med. 21:248-250.

Palmer S.A. 1972. Toxicity of 45 organic herbicides to cattle, sheep and chickens. USDA-ARS Prod. Res. Rep. 137:22-23.

Plumlee F.B. 2006. Metais e outros compostos inorgânicos, p.1637-1640. In: Smith B.P. (Ed.), Medicina Interna de Grandes Animais. 3a-ed. Manole, Barueri.

Radostits O.M., Gay C.C., Blood D.C., Hinchcliff K.W. \& Peter D. Constable 2007. Veterinary Medicine: A textbook of the diseases of cattle, horses, sheep, pigs and goats. $10^{\text {th }}$ ed. W.B. Saunders, Philadelphia. 2065p.

Rehner G.I. 2003. Arsenic/requirements and toxicology, p.311-316. In: Caballero B. (Ed.), Encyclopedia of Food Sciences and Nutrition. Elsevier, Philadelphia.

Riet-Correa F. 2007. Intoxicação por arsênico, p.50-51. In: Riet-Correa F., Schild A.L., Lemos R.A.A. \& Borges J.R.J. (Eds), Doenças de Ruminantes e

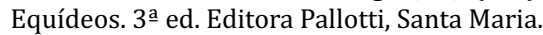

Selby L.A., Case A.A., Osweiler G.D. \& Hayes Jr H.M. 1977. Epidemiology and toxicology of arsenic poisoning in domestic animals. Environ. Health Perspect. 19:183-189.

Valentine B.A., Rumbeiha W.K., Hensley T.S. \& Halse R.R. 2007. Arsenic and metaldehydetoxicosis in a beef herd. J. Vet. Diagn. Invest. 19:212215. 